Check for updates

Cite this: Phys. Chem. Chem. Phys., 2017, 19, 15842

Received 10th February 2017, Accepted 22nd May 2017

DOI: $10.1039 / c 7 c p 00900 c$

rsc.li/pccp

\title{
Extra electronic outer-shell peculiarities accessible under a joint XPS and DFT study $\dagger$
}

\author{
A. R. Cholach, (D *a I. P. Asanov, ${ }^{\text {bc }}$ A. A. Bryliakova ${ }^{a}$ and A. V. Okotrub ${ }^{\text {bc }}$
}

\begin{abstract}
Electronic configuration of chemically bound atoms at the surface, including adsorbed species, or in the bulk of a solid contains a set of natural traps for energy absorption provided by valence band transitions or plasmon oscillations. The core level excitation of any origin is generally coupled with those traps, forming a multichannel route for nonradiative energy dissipation. Using an example of Pt and graphitebased materials, the study shows experimental tracing over these channels by means of elastic electron scattering and X-ray photoelectron spectroscopy. As a complement to the experimental data, calculations of the density of states provide information on chemical behavior and local geometry of the atoms in a sample.
\end{abstract}

\section{Introduction}

Intrinsic properties of a sample can be fortunately exposed in specific responses to a core-level excitation. For example, the following effects have been detected and have become the basis of advanced techniques under the tuneable synchrotron irradiation: X-ray absorption fine structure (XAFS), resonant inelastic X-ray scattering (RIXS), resonant photoemission (RPES) and Auger electron (RAES) spectroscopies. ${ }^{1}$ The XAFS appears both near the photoionization threshold (NEXAFS) and in the extended region far beyond the absorption edge (EXAFS) and reveals the vacant state structure and local geometry, respectively, of the sample. ${ }^{2}$ The RIXS, which is enabled by the energy and momentum transferred by a photon near the absorption edge, exhibits the intrinsic excitations of the material. ${ }^{3}$ RPES and RAES investigate the local electronic structure and electron correlations in a system, respectively. ${ }^{4}$

Similar effects were also discovered under the electron impact as another source of the resonant core-level excitation: ${ }^{5,6}$ a fine structure above the threshold in elastic scattering spectra, which corresponds to the valence state structure of adsorbed species, substrate atoms, and plasmon oscillations. Experimental evidence for conjugate electron excitation (CEE) occurring in ever-present satellites is obtained in the Disappearance Potential spectra from

\footnotetext{
${ }^{a}$ Boreskov Institute of Catalysis, Akademik Lavrentiev Ave 5, Novosibirsk 630090, Russian Federation. E-mail: cholach@catalysis.ru

${ }^{b}$ Nikolaev Institute of Inorganic Chemistry, Akademik Lavrentiev Ave 3, Novosibirsk 630090, Russian Federation

${ }^{c}$ Novosibirsk State University, Pirogov Str 2, Novosibirsk 630090,

Russian Federation

$\dagger$ Electronic supplementary information (ESI) available: Optimized atomic coordinates, density of states and relevant views for the $\mathrm{C}_{24}, \mathrm{C}_{24} \mathrm{~F}_{12}$ and $\mathrm{C}_{24} \mathrm{~F}_{12} \mathrm{Br}_{2}$ unit cells are available in ref. 26. See DOI: 10.1039/c7cp00900c
}

various adsorbed layers, while theoretical evidence is ground in the CEE mechanism, which occurs in a combination of wellknown electron transitions. Indeed, shake-up plasmons are usual objects in X-ray Photoelectron (XPS) and Auger Electron Spectroscopies (AES). ${ }^{7,8}$ The valence band ionization of comparable efficiency for substrate atoms and adsorbed species is the basis of Ultraviolet Photoelectron Spectroscopy (UPS). ${ }^{9}$ CEE replicas above different thresholds in DAPS spectra confirm that core levels are identical with regard to the excitations. ${ }^{5,6}$ Therefore, the CEE phenomena form a multichannel route for nonradiative energy dissipation within the DAPS probing depth of 2-3 monolayers (ML) and do not break the general concepts in the field of electron-solid interaction. The present study verifies similar effects in the bulk of model graphite-based samples under the non-resonant X-ray core level excitation.

\section{Experimental}

The Disappearance Potential spectroscopy (DAPS) is based on the threshold core-level excitation of a target atom by an electron beam with time-based energy $E_{\mathrm{p}} \cdot{ }^{10}$ Whenever an accelerating potential overcomes the core level energy, a part of scattered electrons disappears from the elastic current $I$, providing a spectral dip in the dependence of $\mathrm{d} I\left(E_{\mathrm{p}}\right) / \mathrm{d} E_{\mathrm{p}}$ on $E_{\mathrm{p}}$. The spectrum appears at $E_{\mathrm{p}}$ equal to the difference between the core and the vacant state energy, while its shape is determined by the self-convolution of the vacant density of states (DOS), to which the core and primary electrons move. Adsorption of gases was performed at $300 \mathrm{~K}$ on the Auger-clean $\mathrm{Pt}(100)-(1 \times 1)$ surface obtained by an NO receipt; ${ }^{11}$ exposures are in Langmuir units ( $1 \mathrm{~L}=10^{-6}$ Torr $\left.\mathrm{s}\right)$. The Fermi level $E_{\mathrm{F}}$ in DAPS spectra corresponds to the incident electron energy $314.8 \mathrm{eV}$ close to 
the $\mathrm{Pt} 4 \mathrm{~d}_{5 / 2}$ eigenvalue. ${ }^{12}$ Other experimental details and methods of spectrum treatment can be found elsewhere. ${ }^{5,6}$

The XPS studies were performed on a Phoibos 150 SPECS spectrometer using monochromatized $\mathrm{Al} \mathrm{K}$ radiation $(1486.7 \mathrm{eV})$. Other experimental details, the low-temperature synthesis technique, and characterization of highly orientated pyrolytic graphite (HOPG), pristine and fluorinated $\mathrm{C}_{2} \mathrm{~F}$ can be found elsewhere. ${ }^{13,14}$ The $\mathrm{Br}_{2}$ embedding into $\mathrm{C}_{2} \mathrm{~F}$ was performed as in ref. 15 and resulted in the $\sim \mathrm{C}_{2} \mathrm{FBr}_{0.15}$ stoichiometric composition.

\section{Theoretical}

Geometric parameters and DOS of used unit cells were calculated within the Density Functional Theory (DFT) using the Quantum Espresso software ${ }^{16}$ and the non-local exchange-correlation functional in the Perdew-Burke-Ernzerhof parameterization. ${ }^{17}$ The interactions between ionic cores and electrons were described by the Projected Augmented Wave (PAW) method ${ }^{18}$ with the kinetic energy cutoff $E_{\text {cut }}=40$ Ry (320 Ry for the charge-density cutoff) for a plane-wave basis set. The Gaussian spreading for the Brillouin-zone integration was 0.02 Ry; the Marzari-Vanderbilt cold smearing was used. ${ }^{19}$ Pure graphite was modeled with a two-layer slab using a $\mathrm{C}_{24}$ unit cell with the optimized lattice parameters $a=2.46 \AA \times 3$ and $b=2.46 \AA \times 2$. Half-fluorinated graphite $\mathrm{C}_{2} \mathrm{~F}$ was modeled with a two-layer slab using a $\mathrm{C}_{24} \mathrm{~F}_{12}$ unit cell with the optimized lattice parameters $a=2.51 \AA \times 3$ and $b=2.46 \AA \times 2$, where $\mathrm{F}$ is attached to $\mathrm{C}$ atoms on both sides of the slab. DFT calculations have found this structure to be energetically most favourable ${ }^{20}$ whereas experimental data indicate another $\mathrm{C}_{2} \mathrm{~F}$ configuration. ${ }^{21}$ Moreover, the common feature of each phase is the presence of fluorinated and unfluorinated carbons, which determines the DOS structure in similar energy regions. The empty space of $40 \mathrm{Bohr}$ in the $Z$-direction was accepted to neglect the interaction between the slabs. The Brillouin zone was integrated on a $20 \times 20 \times 1$ grid of Monkhorst-Pack $k$-points. ${ }^{22}$ The accuracy was verified by testing the energy convergence. The default number of bands (NB) for the $\mathrm{C}_{24}$ unit cell is shown to result in a DOS structure that is close to the relevant data on graphite and graphene. ${ }^{23-26}$ However, in this study, we accepted $\mathrm{NB}=384$ and 288 for $\mathrm{C}_{24}$ and $\mathrm{C}_{24} \mathrm{~F}_{12}$ unit cells, respectively; these larger NBs preserve the structure of the occupied valence band (VB) and enable a larger vacant DOS energy, providing better agreement between the model and the experimental data. DFT calculations were performed for the unit cells with the Bernal and hexagonal (Hex) structures. ${ }^{20}$ Trial DFT runs for the Hex $\mathrm{C}_{24}$ unit cell have not revealed a perceptible DOS change under the van der Waals (vdW) interaction, except a slight change in $\mathrm{p}_{z} \cdot{ }^{26}$ The atomic composition of unit cells $\mathrm{C}_{24} \mathrm{~F}_{12} \mathrm{Br}_{2}$ was chosen on the basis of the stoichiometric composition of the sample $\mathrm{C}_{2} \mathrm{FBr}_{0.15}$ used in XPS studies. The work function $\varphi=4.6 \mathrm{eV}^{23}$ was accepted for each of the $\mathrm{C}_{24}$-based unit cells.

The Pt DOS was calculated using the Perdew-Burke-Ernzerhof functional $^{27}$ and the PAW with the optimized lattice constant $3.99 \AA$. In this case, $E_{\text {cut }}=40$ Ry and a $12 \times 12 \times 12$ grid of Monkhorst-Pack $k$-points were applied.

\section{CEE effect in the near-surface layer}

Conventional DAPS theory directs the core and primary electrons to vacant states around $E_{\mathrm{F} .}{ }^{16}$ The larger the spectral dip is, the larger the vacant DOS is, whereas the lack of vacant DOS results in no signal. ${ }^{10}$ Moreover, DAPS is certainly sensitive to other channels of elastic electron consumption related specifically to the CEE phenomena and consists of independent shake-off and shake-up VB transitions, each coupled with the threshold core level excitation of a target atom. These channels of energy dissipation can be approximated as an electron transition from the ground DOS $\sigma_{\mathrm{VB}}$ to the vacant DOS $\sigma_{\mathrm{Vac}}$, whose probability $W(E)$ is in proportion to convolution on the absolute energy relative to $E_{\mathrm{F}}$ with a matrix element $f(E, \sigma)$ :

$$
\begin{aligned}
W_{\mathrm{up}}(E) & =\int_{0}^{E} f(E, \sigma) \sigma_{\mathrm{VB}}(|E|) \sigma_{\mathrm{Vac}}(E-\varepsilon) \mathrm{d} \varepsilon \\
W_{\mathrm{off}}(E) & =f(E, \sigma) \sigma_{\mathrm{VB}}(|E|)
\end{aligned}
$$

The shake-off mode moves $\sigma_{\mathrm{VB}}$ to the vacant DOS at the vacuum level. The cross-section per solid angle for the non-relativistic electron-electron scattering is efficient for small angles $\Theta$ according to the Rutherford relation $\mathrm{d} s / \mathrm{d} \Omega \sim 1 / \sin ^{4}(\Theta / 2){ }^{28,29}$ Therefore, the CEE probability in eqn (1) should include one-dimensional (1D) $\sigma_{\text {Vac }}$ rather than the total free DOS. As demonstrated in Fig. 1(a), Van Hove singularities determine the $1 \mathrm{D}$ DOS to be 0 , infinity, and $1 / \sqrt{E-E_{\mathrm{F}}-\phi}$ at the energies below, at, and above the vacuum level, respectively, ${ }^{30}$ where $\varphi=5.6 \mathrm{eV}$ is a $\operatorname{Pt}(100)$ work function. ${ }^{31}$ This behavior provides the resonant character of CEE and multiple tracing over adsorbed species, including hydrogen atoms and reaction intermediates. ${ }^{5,6}$ A shake-off CEE satellite due to the adsorbed species is a distinct peak with the 1-2 eV base-width and coverage-proportional intensity, located at a given ionization potential above each threshold. The DAPS spectra in Fig. 1 particularly exhibit $1 \pi, 5 \sigma$, and $4 \sigma$ valence states of the adsorbed $\mathrm{CO}$ molecule and the $\sigma$ state of $\mathrm{H}_{\mathrm{ad}}$ species, which fit published UPS data. ${ }^{32-35}$ Similar accordance has been found for adsorbed $\mathrm{O}, \mathrm{N}, \mathrm{NO}$, and $\mathrm{NH}$ particles. ${ }^{6}$
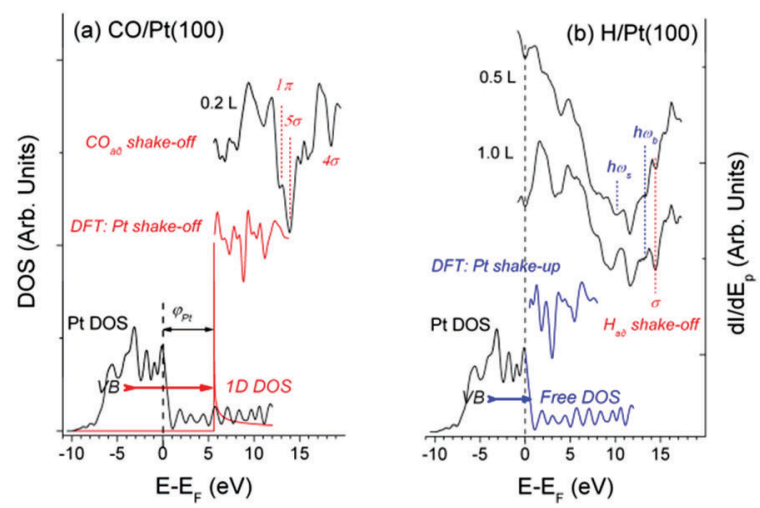

Fig. 1 DAPS spectra obtained after exposure of $\mathrm{Pt}(100)$ surface to (a) CO as compared to simulated shake-off transitions of occupied Pt DOS to the vacuum level (1D DOS) and (b) $\mathrm{H}_{2}$ as compared to shake-up transitions of occupied Pt $d_{z x}+d_{z y}+d_{x y}$ states to the free DOS. 
The theoretical Pt shake-off spectrum in Fig. 1(a) was constructed from DFT calculations via the following sequence: VB inversion (because of $W_{\text {off }}(E)$ in eqn (1) and, therefore, the larger the $\sigma_{\mathrm{VB}}$, the larger the spectral dip), differentiation, and shifting to the higher energy by $\varphi_{\mathrm{Pt}}$. A substantial contribution of the adsorbed layer into DAPS spectra (as compared with substrate atoms) is a result of the exceptional surface sensitivity of the DAPS, whose probing depth is determined by half the electron mean free path in a solid and thus is restricted to 2-3 ML.

The theoretical Pt shake-up spectrum in Fig. 1(b) corresponds to the convolution of the partial $d$ states by eqn (1). The simulated Pt shake-up and shake-off spectra in Fig. 1 are close to each other because of strongly localized vacant states at $E_{\mathrm{F}}$ and 1D DOS at the vacuum level, respectively, and because of the domination of the equal $\mathrm{d}_{z x}, \mathrm{~d}_{z y}$, and $\mathrm{d}_{x y}$ states in the total Pt DOS. ${ }^{26}$ Close similarity between experimental and simulated spectral fragments in Fig. 1 implies regular involvement of $\mathrm{Pt}$ DOS into events, similar matrix elements $f(E, \sigma)$ for different partial densities of states (pDOS) in eqn (1), and no symmetry ban for CEE transitions. Furthermore, Fig. 1(b) traces adsorbed hydrogen atoms, which is not available for AES and XPS. It is worth noting that none of the CEE satellites in the DAPS spectra could be assigned to the transition from the valence band of adsorbed species to the vacant state of the adjacent substrate atom. ${ }^{5,36}$ In contrast to VB CEE features, the surface plasmon disappears while the bulk plasmon decreases on coverage, as shown in Fig. 1(b), due to screening by the adsorbed layer. ${ }^{5}$ Multiple plasmon excitations are also detected at energy points aliquot to the bulk plasmon energy in Fig. 1(b). ${ }^{5}$ The agreement between the calculated and experimental data in Fig. 1 confirms that both vacancies, at $E_{\mathrm{F}}$ and at the vacuum level, are appropriate spots for the allocation of excited electrons under CEE transitions. Present examination accentuates the generality of the CEE effect under inelastic electron scattering by any adsorbed system, bringing to light the outer shell structure of near-surface atoms, while the Auger effect discloses the inner shell peculiarities. CEE control seems to be an additional tool of a standard electron analyzer for fingerprinting the adsorbed layer at the atomic-molecular level and an alternative to the RPES, which requires tunable synchrotron irradiation and fine analytical instrumentation. ${ }^{4}$ In addition, the elastic electron scattering basically provides the vacant state structure and geometrical parameters, similar to NEXAFS and EXAFS, respectively. ${ }^{37}$ In prospect, the CEE control should empower the localization of adsorbed species over a composite surface. Indeed, relevant satellites accompany the threshold excitation of only such a surface atom that is chemically bound to the adsorbed particle, while core level energies are easily distinguishable. The following CEE regularities are thus verified.

- Shake-up transitions include ground and excited states of the same atom and probably have no symmetry prohibition.

- Shake-off excitations are available for both the substrate atom and the adsorbed species, although the former is the only over-energy carrier. The infinite free 1D DOS at the vacuum level is a common spot of VB destination. There is no symmetry ban for shake-off transitions, whose satellite structure above the threshold is a $\sigma_{\mathrm{VB}}$ mirror-image with respect to $E_{\mathrm{F}}$ in eqn (1) shifted to higher energy by the work function.

- The plasmon oscillation is evidence for the collective CEE of free electrons.

\section{CEE effect in the bulk of a solid}

CEE phenomena have been successfully traced within the DAPS probing depth of 2-3 ML. The fundamental affinity between the electronic structure of near-surface and bulk atoms assumes similar channels of electron energy dissipation accompanying the core level excitation, irrespective of it occurring by primary electrons or X-ray irradiation. Therefore, the photoelectron can lose a part of its energy for CEE processes and result in a fine XPS spectral structure providing the outer shell peculiarities. By analogy with DAPS measurements, CEE in a multicomponent substance under the non-resonant X-ray core level excitation should include the following:

- Shake-off VB transitions to the vacuum level, where pDOS should be considered through possible differences in the matrix elements in eqn (1). The same ground state (VB), the common point of destination (1D DOS), and enough energy excess of any photoelectron, a former core electron, should result in analogous energy losses in XPS spectra of different target components.

- Shake-up VB transitions to free states, with the preferable convolution of the pDOS of the same atom. The unified VB of chemically bound atoms probably gives no preference for a photoelectron to detach the energy for a particular CEE transition. Then, similar fine structures are again expected in XPS spectra of different sample components.

- Plasmon oscillations, if any.

\section{XPS HOPG vs. DFT $\mathrm{C}_{24}$ unit cell}

DFT runs revealed that the DOSs obtained for the $\mathrm{C}_{24}$ unit cells with the Bernal and Hex structure are similar and close to the DOS of graphite/graphene (Fig. 2(a and b)). The optimized C-C bond length $d_{\mathrm{C}-\mathrm{C}}=1.42 \AA$ fits to that of graphite, whereas the interlayer distance $d_{\text {layer }}=3.96 \AA$ is somewhat larger $(3.35 \AA$ for graphite), which could be expected for the bilayer unit cells without vdW interaction. Accounting for the vdW interaction has resulted in $d_{\text {layer }}=3.34 \AA$ for the Hex $\mathrm{C}_{24}$ unit cell. It has also led to the negligible change in DOS as compared with Fig. 2(a) and, therefore, to the same (as in Fig. 2(c)) CEE transitions that are responsible for the energy losses. The current study focuses on the DOS structure, whose similarity makes it admissible to confront XPS and DFT data related to bulk material and a suitable unit cell, respectively, omitting the vdW correlation.

Conventional satellites at higher energy sides of XPS spectra truly indicate the photoelectron energy consumption. The CEE approach enables complete description of the HOPG XPS C1s spectrum by the combination of shake-up and shake-off transitions (Fig. 2(c)). The matrix elements $f(E, \sigma)$ in eqn (1) were accepted to be unity for a $W(E)$ basis set, while the Y-scale magnification in Fig. 2(c) evaluates $f(E, \sigma)$ as a contribution of a 

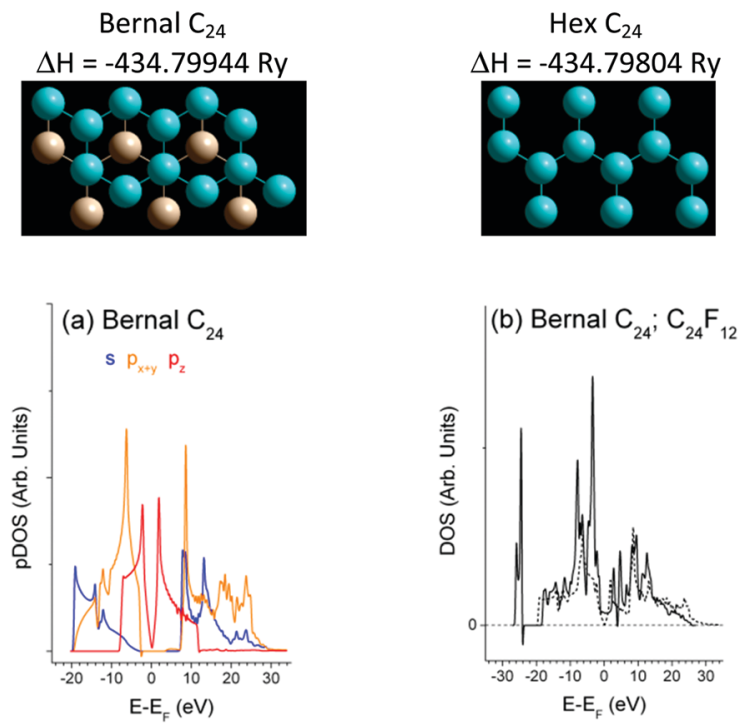

(c) HOPG C1s \& $\mathrm{C}_{24}$ Unit Cells

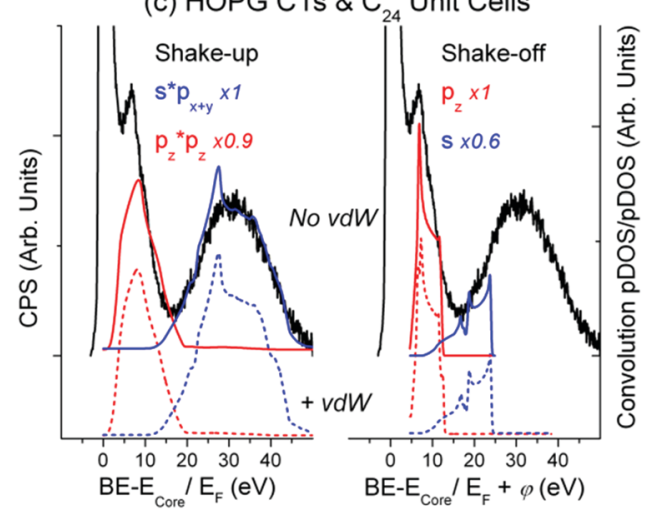

Fig. 2 (a) Partial DOS of the $C_{24}$ unit cell $\left(p_{x}=p_{y}\right)$; (b) total DOS of $C_{24}$ (dashed line) and $\mathrm{C}_{24} \mathrm{~F}_{12}$ unit cells; (c) energy losses above the core level energy $(284.6 \mathrm{eV})$ in a HOPG XPS C1s spectrum as compared to CEE transitions in the Bernal and Hex $\mathrm{C}_{24}$ unit cell omitting (solid lines) and accounting for (dashed lines) the van der Waals interaction, respectively; top panel shows relevant $X-Y$ views and the formation energies $(\Delta H)$.

particular CEE transition into calculated energy consumption to fit the experimental photoelectron energy losses. As in the case of Pt (Fig. 1), the shake-off probability $W(E)_{\text {off }}$ is a $\sigma_{\mathrm{VB}}$ mirror-image with respect to $E_{\mathrm{F}}$ shifted by $\varphi$ to higher energy. The satellite $\sim 5.5 \mathrm{eV}$ in Fig. 2(c) is usually assigned to a $\pi$ plasmon responsible for the $\pi \rightarrow \pi^{*}$ transition, ${ }^{38}$ although the classic plasmon stands for collective oscillations of the free electrons missing in HOPG according to Fig. 2(b) and ref. 24. Moreover, this satellite rather originates from shake-off than from shake-up $\mathrm{p}_{z}$ transition (Fig. 2(c)).

\section{XPS $\mathrm{C}_{2} \mathrm{~F}$ vs. DFT $\mathrm{C}_{24} \mathrm{~F}_{12}$}

As seen from Fig. 3, there is expected similarity between higher energy tails of ${ }^{(\mathrm{F})} \mathrm{C} 1 \mathrm{~s}$ (C is bound to $\mathrm{F}$ atom) and F1s XPS spectrum, in which the background of multiple external and surface energy losses has been subtracted according to ref. 39 .

Fine XPS spectral structures above $10 \mathrm{eV}$ conform well to shake-up (Fig. 3(a)) and shake-off (Fig. 3(b)) transitions.

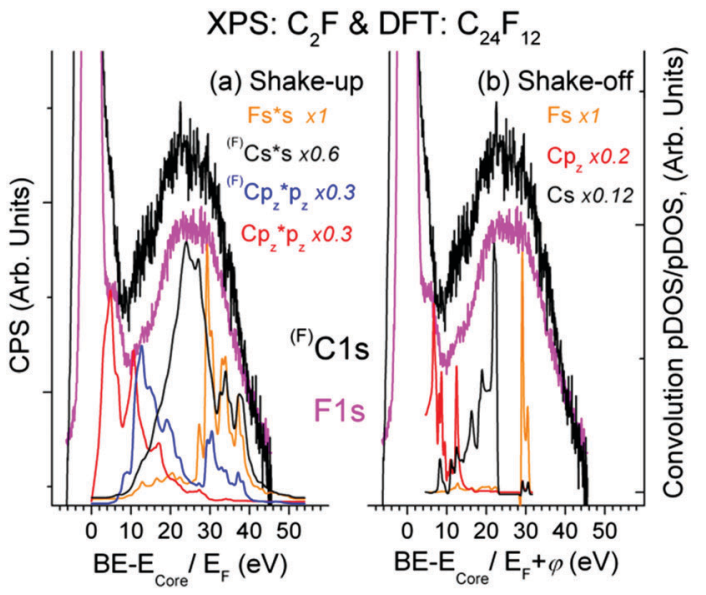

Fig. 3 Shake-up (a) and shake-off (b) transitions in the Bernal $\mathrm{C}_{24} \mathrm{~F}_{12}$ unit cell in comparison with the XPS C1s spectrum and the XPS F1s spectrum of $\mathrm{C}_{2} \mathrm{~F}$. The $\mathrm{C} 1 \mathrm{~s}$ and $\mathrm{F} 1 \mathrm{~s}$ spectra are normalized to the intensity of the main peak (at $E_{\text {Core }}=287.6 \mathrm{eV}$ and $687.4 \mathrm{eV}$, respectively).

The feature $\sim 5.4 \mathrm{eV}$ is usually assigned to conjugated $\pi$ bonds in a chain of $\mathrm{C}$ atoms; ${ }^{14}$ it fits the shake-up $\mathrm{Cp}_{z}$ transition and naturally accompanies the C1s spectrum since the $\pi$ bond is localized exclusively at the $\mathrm{Csp}^{2}$ atom, not bound to F. Finding a similar satellite in the F1s spectrum is rather remarkable from the viewpoint of classical theory (because $\mathrm{F}$ atoms are not involved in the $\pi$ bond), but is quite in line with the CEE model. The base line shift in Fig. 3 relative to $\mathrm{C} 1 \mathrm{~s}=285.1 \mathrm{eV}$ (C is not bound to F) does not contradict the DFT data, but enables the other shake-off transitions $\left({ }^{(\mathrm{F})} \mathrm{Cp}_{y}, \mathrm{Fp}_{y}, \mathrm{Fp}_{z}\right)$ to contribute to the $\sim 5.4 \mathrm{eV}$ feature. The formation energy of the Bernal unit cell is by $0.008 \mathrm{eV}$ higher than that of the Hex $\mathrm{C}_{24} \mathrm{~F}_{12}$ cell, while pDOSs of both structures are very similar. A sizable DOS at $E_{\mathrm{F}}$ shown in Fig. 2(b) accepts plasmon oscillations that can give the energy loss at 9.1 or $12.9 \mathrm{eV}$ for 1 or 2 free electrons per a $\mathrm{C}_{2} \mathrm{~F}$ fragment, respectively.

\section{XPS $\mathrm{C}_{2} \mathrm{~F}+\mathrm{Br}_{2}$ vs. DFT $\mathrm{C}_{24} \mathrm{~F}_{12} \mathrm{Br}_{2}$}

Partial DOS of the pristine $\mathrm{C}_{24} \mathrm{~F}_{12}$ unit cell in Fig. 4(a) undergoes little change in comparison with that of $\mathrm{C}_{24} \mathrm{~F}_{12} \mathrm{Br}_{2}$ in Fig. 4(b). The XPS spectra in Fig. 5(a), obtained after the $\mathrm{Br}_{2}$ intercalation, correspond to the stoichiometric composition $\mathrm{C}_{2} \mathrm{FBr}_{0.15}$ and reveal the lack of new features as compared with Fig. 3. Energy losses in the F1s spectrum are less pronounced than those in C1s; however, both spectra are in agreement with the same CEE transitions as before the $\mathrm{Br}_{2}$ embedding. The Bromine $\mathrm{p}_{z^{-}}$and $\mathrm{s}$ - shake-off transition conforms well to the non-resolved $\sim 9.5 \mathrm{eV}$ peak and the $\sim 20 \mathrm{eV}$ shoulder in Fig. 5(a), respectively. The higher energy part of the XPS Br3d spectrum is similar to that of F1s and C1s, but it does not correspond to any CEE within Br pDOS. This probably indicates a bonding between a $\mathrm{Br}_{2}$ molecule and the $\mathrm{C}_{2} \mathrm{~F}$ frame that is enough to provide the Br3d photoelectron energy losses via the CEE transitions in pDOS of $\mathrm{C}$ and $\mathrm{F}$.

The difference F1s spectrum in Fig. 5(b) exhibits a distinct structure, which conforms to shake-up transitions of the pDOS 

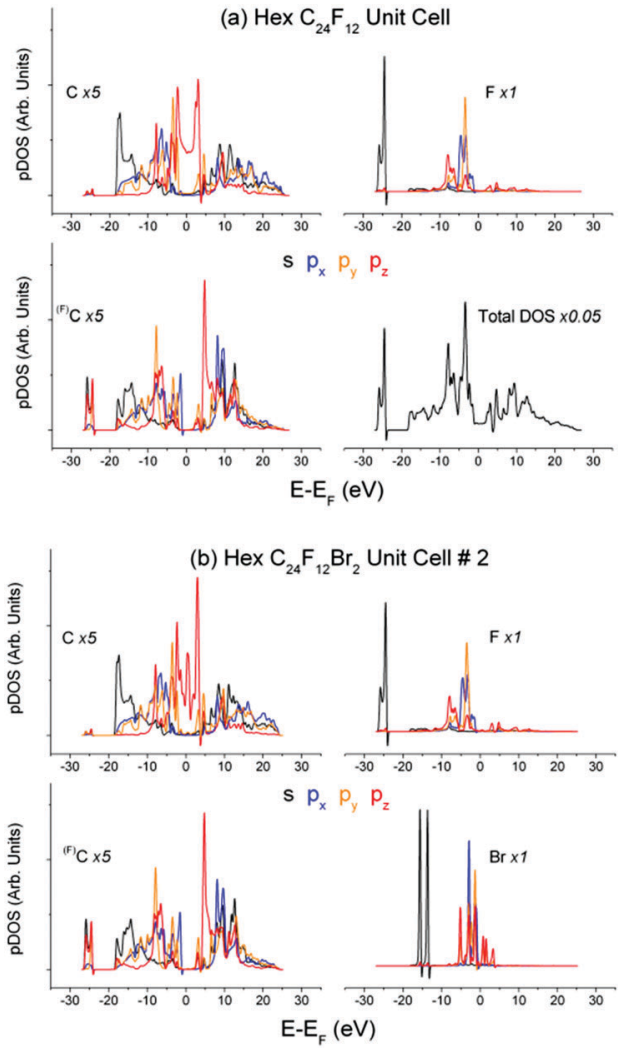

Fig. 4 Partial DOS of (a) $\mathrm{C}_{24} \mathrm{~F}_{12}$ and (b) $\mathrm{C}_{24} \mathrm{~F}_{12} \mathrm{Br}_{2}$ unit cell No. 2
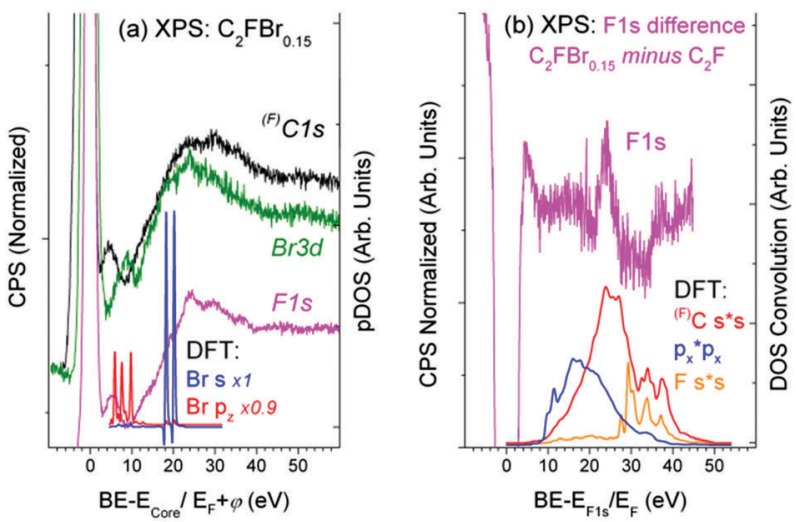

Fig. 5 (a) XPS $C 1 s, F 1 s$, and $B r 3 d$ spectra for $\mathrm{Br}_{2}$-intercalated $\mathrm{C}_{2} \mathrm{~F}$ (relative respectively to $E_{\text {Core }}=288.6,687.2$, and $70.7 \mathrm{eV}$ ) in comparison with the shake-off transitions in the $\mathrm{C}_{24} \mathrm{~F}_{12} \mathrm{Br}_{2}$ unit cell No. 2; (b) difference F1s spectrum as compared with the shake-up transitions in the same unit cell.

responsible for $\mathrm{C}-\mathrm{F}$ bonding, which can be interpreted as the $\mathrm{C}-\mathrm{F}$ bond strengthening. It seems reasonable because $\mathrm{Br}_{2}$ embedding weakens the interactions between carbon layers, which should be accompanied with the balanced enrichment of the occupied DOS of C and F. The difference XPS C1s spectrum shows similar, but less pronounced, features (Fig. 5(b)).

The DFT studies were performed for the Bernal and Hex $\mathrm{C}_{24} \mathrm{~F}_{12} \mathrm{Br}_{2}$ unit cells $\# 1-4$ at the extreme entry angles $\alpha_{0}=0^{\circ}$ and $\alpha_{0}=90^{\circ}$ between the $\mathrm{Br}-\mathrm{Br}$ axis and carbon planes (Table 1 ).
Table 1 Parameters of the $\mathrm{C}_{24} \mathrm{~F}_{12} \mathrm{Br}_{2}$ unit cells: entry $\alpha_{0}$ and optimized $\alpha$ angles between $\mathrm{Br}-\mathrm{Br}$ axis and carbon planes; $\mathrm{Br}-\mathrm{Br}$ bond length $d_{\mathrm{BrBr}}$ and interlayer distance $d_{\text {layer; }}$ weighted average $\mathrm{Br} s-\left\langle E_{\mathrm{s}}\right\rangle$ and $\mathrm{p}-\left\langle E_{\mathrm{p}}\right\rangle \mathrm{DOS}$ relative $E_{\mathrm{F}}$, the difference $\Delta_{\mathrm{s}-\mathrm{p}}=\left\langle E_{\mathrm{p}}\right\rangle-\left\langle E_{\mathrm{s}}\right\rangle$ and relative formation energy $\Delta H$; Deviation of $\Delta_{\mathrm{s}-\mathrm{p}}$ in a unit cell from that of separate species

\begin{tabular}{llllll}
\hline Parameter & $\# 1$ & $\# 2$ & $\# 3$ & $\# 4$ & $\# 5$ \\
\hline$\alpha_{0}$ & $0^{\circ}$ & $90^{\circ}$ & $0^{\circ}$ & $90^{\circ}$ & $52^{\circ}$ \\
$\alpha^{a}$ & $0.2^{\circ}$ & $23.7^{\circ}$ & $6.2^{\circ}$ & $0.1^{\circ}$ & $0.7^{\circ}$ \\
& $2.4^{\circ}$ & $24.2^{\circ}$ & $8.9^{\circ}$ & $9.9^{\circ}$ & $0.9^{\circ}$ \\
& & $50.8^{\circ}$ & $20.2^{\circ}$ & $24.2^{\circ}$ & $22.4^{\circ}$ \\
& & $78.0^{\circ}$ & & $31.4^{\circ}$ & $29.9^{\circ}$ \\
$d_{\text {BrBr }}(\AA)$ & 3.24 & 2.45 & 2.37 & 3.18 & 3.34 \\
$d_{\text {layer }}(\AA)$ & 4.88 & 6.74 & 7.38 & 5.70 & 5.11 \\
& 6.32 & 8.21 & 7.39 & 5.98 & 5.82 \\
& & & & 6.02 & 6.74 \\
$\left\langle E_{\mathrm{s}}\right\rangle(\mathrm{eV})$ & -13.24 & -14.61 & -15.08 & -15.34 & -15.10 \\
$\left\langle E_{\mathrm{p}}\right\rangle(\mathrm{eV})$ & -1.58 & -2.55 & -2.75 & -2.66 & -2.49 \\
$\Delta_{\mathrm{s}-\mathrm{p}}(\mathrm{eV})$ & 11.66 & 12.06 & 12.32 & 12.68 & 12.61 \\
$\Delta H(\mathrm{eV})$ & 0 & 0.79 & 0.42 & 1.28 & 0.74 \\
& & & & & \\
$\Delta_{\mathrm{s}-\mathrm{p}}$ Deviation & $(\mathrm{eV})$ & & & & \\
$\mathrm{Br}_{2}^{0}$ & 1.39 & 0.99 & 0.73 & 0.37 & 0.44 \\
$\mathrm{Br}_{2}{ }^{-1}$ & 0.4 & 0 & -0.26 & -0.62 & -0.55 \\
$\mathrm{Br}_{1}^{0}$ & 0.62 & 0.22 & -0.04 & -0.4 & -0.33 \\
$\mathrm{Br}_{1}{ }^{-1}$ & 0.07 & -0.33 & -0.59 & -0.95 & -0.88
\end{tabular}

${ }^{a}$ Each value corresponds to $\mathrm{C}$ triplet representing the plane. ${ }^{26}{ }^{b} \mathrm{~A}$ set answers the zigzag arrangement of carbon planes; compare to $d_{\text {layer }}=$ $4.08 ; 5.52$ and $3.75 ; 3.98 \AA$ for the Hex and Bernal $\mathrm{C}_{24} \mathrm{~F}_{12}$ unit cell, respectively. ${ }^{26}$

The calculations revealed that the $\mathrm{Br}_{2}$ embedding enlarges the interlayer distance, but insignificantly affects the pDOS of $\mathrm{C}$ and $\mathrm{F}$. The latter conforms to the chemical inertness of the pristine $\mathrm{C}_{2} \mathrm{~F}$ cell and to the low $\mathrm{Br}$ content in the product substance $\mathrm{C}_{2} \mathrm{FBr}_{0.15}{ }^{40}$ Another DFT run for Bernal unit cell \#5 at $\alpha_{0}=52^{\circ}$ (about optimal $\alpha$ for Hex \#2) resulted in new optimized parameters in Table 1. Both atomic (No. 1, 4 and 5) and molecular (No. 2 and 3 in Table 1) states of intercalated $\mathrm{Br}_{2}$ are available, and each of the local structures seems appropriate. No preference can be given to a particular case at this phase of the conventional DFT study. Moreover, each of the five unit cells is characterized by the specific Br pDOS structure (Fig. 6). Further processing was attempted to choose the appropriate cell out of the five unit cells in Table $1 . \$$

First, DFT examinations of separate species revealed a pronounced difference $\Delta_{\mathrm{s}-\mathrm{p}}=0.2-1.3$, which is far beyond the accuracy of calculations $(0.01 \mathrm{eV})$, between the weighted average energy $\left\langle E_{\mathrm{s}, \mathrm{p}}\right\rangle$ of the Br s- and p-DOS (Table 2). The energy $\left\langle E_{\mathrm{s}, \mathrm{p}}\right\rangle$ was determined as $E, \sum \sigma_{i} E_{i} / \sum \sigma_{i}$, and middle of $\int \sigma_{i} \mathrm{~d} E$ for the $\mathrm{Br}$ atom with single, several localized, and diffused s- and p-DOS, respectively.

Second, the empirical parameter $\Delta_{\mathrm{s}-\mathrm{p}}$ was taken as a descriptor of the Br state, in the same way as the apparent binding energy determines the oxidation rate in XPS practice. The smallest deviations $\Delta_{\mathrm{s}-\mathrm{p}}$ of $0.02,0$, and $0.04 \mathrm{eV}$ make cells $\# 1,2$, and 3, preferable, respectively (Table 1 ). Third, in the case of the large distance $d_{\mathrm{BrBr}}$ (the $\mathrm{Br}-\mathrm{Br}$ bond is lost), the difference

$\ddagger$ A similar processing may be useful for identification of the state of the atoms in other compounds whereas its theoretical rationalizing is beyond this paper. 


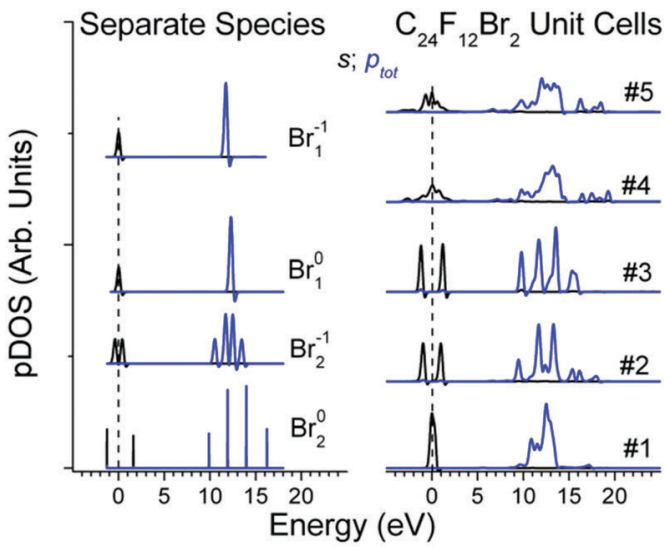

Optimized $\mathrm{C}_{24} \mathrm{~F}_{12} \mathrm{Br}_{2}$ unit cell \#2

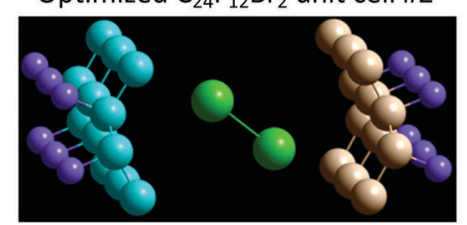

Fig. $6 \mathrm{Br}$ s- and a sum of $p_{x}+p_{y}+p_{z}$ DOS for the separate species and $\mathrm{C}_{24} \mathrm{~F}_{12} \mathrm{Br}_{2}$ unit cells; $X$ scales are aligned by the average of the $\mathrm{Br}$ s-DOS in $\mathrm{Br}_{2}$ molecule. Lower panel shows the optimal unit cell $\mathrm{C}_{24} \mathrm{~F}_{12} \mathrm{Br}_{2}$ No. 2 out of appropriate DFT runs.

Table 2 Weighted average $\mathrm{Br} \mathrm{s}-\left\langle E_{\mathrm{s}}\right\rangle$ and $\mathrm{p}-\left\langle E_{\mathrm{p}}\right\rangle$ DOS relative $E_{\mathrm{F}}$, the difference $\Delta_{\mathrm{s}-\mathrm{p}}=\left\langle E_{\mathrm{p}}\right\rangle-\left\langle E_{\mathrm{s}}\right\rangle$ and $\mathrm{Br}-\mathrm{Br}$ bond length $d_{\mathrm{BrBr}}$ in separate species

\begin{tabular}{lllll}
\hline$(\mathrm{eV})$ & $\mathrm{Br}_{2}^{0}$ & $\mathrm{Br}_{2}{ }^{-1}$ & $\mathrm{Br}_{1}^{0}$ & $\mathrm{Br}_{1}{ }^{-1}$ \\
\hline$\left\langle E_{\mathrm{s}}\right\rangle$ & -20.49 & -13.43 & -12.37 & -11.91 \\
$\left\langle E_{\mathrm{p}}\right\rangle$ & -7.44 & -1.37 & -0.09 & -0.18 \\
$A_{\mathrm{s}-\mathrm{p}}$ & 13.05 & 12.06 & 12.28 & 11.73 \\
$d_{\mathrm{BrBr}}(\AA)$ & 2.29 & 2.85 & - & -
\end{tabular}

$\Delta_{\mathrm{s}-\mathrm{p}}$ in a cell should be close to that of separate $\mathrm{Br}_{1}$. Otherwise (i.e. the $\mathrm{Br}-\mathrm{Br}$ bond retains), the difference $\Delta_{\mathrm{s}-\mathrm{p}}$ should be close to that of $\mathrm{Br}_{2}$ species. In this case, $d_{\mathrm{BrBr}}$ for cell $\# 1$ (3.24 $\AA$ ) and for cell \#2 (2.45 ̊) corresponds to the lack of the $\mathrm{Br}-\mathrm{Br}$ bond in $\mathrm{Br}_{1}{ }^{-1}$ and to $d_{\mathrm{BrBr}}$ in $\mathrm{Br}_{2}^{0}(2.29 \AA)$ ), respectively (Table 1$)$.

On the contrary, cell \#3 should be ruled out because its $d_{\mathrm{BrBr}}=2.37 \AA$ implies that the $\mathrm{Br}-\mathrm{Br}$ bond is retained, while the $\mathrm{Br}_{1}^{0}$ specimen with nearest $\Delta_{\mathrm{s}-\mathrm{p}}$ has no bond. Finally, cell \#2 is preferred over cell $\# 1$ with respect to the formation energy (Table 1). In addition, the reaction $\mathrm{C}_{24} \mathrm{~F}_{12}+\mathrm{Br}_{2} \rightarrow \mathrm{C}_{24} \mathrm{~F}_{12} \mathrm{Br}_{2}$ is found endothermic for cell \#1 in contrast to others. ${ }^{26}$

The optimal unit cell (cell \# 2, Fig. 6) corresponds to the enlargement of the interlayer distance by 2-4 $\AA$ under $\mathrm{Br}_{2}$ embedding into $\mathrm{C}_{24} \mathrm{~F}_{12}$ and to the angles $24-78^{\circ}$ between the $\mathrm{Br}-\mathrm{Br}$ axis and various planes of the C-skeleton. These results agree well with experimental X-ray absorption studies that have reported the angles $20^{\circ}-30^{\circ}$ and the molecular $\mathrm{Br}_{2}$ state for similar systems. ${ }^{15,41}$

The CEE control by the joint XPS and DFT efforts can be characterized by the following obvious, verified or probable resources.
- The comparison of extended XPS spectra with theoretical CEE transitions can provide the local geometry and bond types in a sample with the use of conventional DFT facilities.

- The occurrence and consequence of bonding between atoms can be enabled because the core level excitation of a given atom is accompanied by CEE satellites of the next one only within the integrated valence band. Examination of the adsorbed layer and strong metal-support interaction at grazing incident can also be promising.

- The multiple CEE control (around various XPS peaks) should improve the reliability of data interpretation, while individual sets of the core level energies facilitate the study of multicomponent materials.

- Similarly to DAPS data, ${ }^{5}$ hydrogen tracing as a CEE satellite above the core level energy of the other sample component does not contradict XPS principles.

- The energy loss spectrum is linked up to the XPS peak and does not depend on the apparent core level energy; therefore, CEE control is available for samples of any conductivity.

- The valence band structure in XPS spectra, being affected by the primary irradiation, differs from that obtained by nondestructive or theoretical methods. Currently, the VB does not undergo the incident X-ray impact, but absorbs a part of photoelectron energy necessary for the resonant transition; therefore, CEE control is a non-destructive method.

- The extensive use of XPS and DFT techniques makes the CEE analysis accessible.

Regular plasmons in AES and XPS spectra, as well as assignment of XPS, RPES and NEXAFS spectra in terms of $\pi, \sigma \rightarrow \pi^{*}$, $\sigma^{*}$ or the Highest Occupied $\rightarrow$ the Lowest Unoccupied Molecular Orbital transitions, ${ }^{42,43}$ are obvious CEE manifestations. The energy dissipation, which follows the core level excitation, is the general trend of electron systems. Auger electron transitions are particular cases of such an event which results from filling the core hole. The CEE phenomena form another natural route of relaxation-by the photoelectron energy redistribution through the valence band. It is worth noting that CEE events are enabled because the former core and VB electrons belong to the same configuration and therefore can be hardly detectable by electron energy loss spectroscopy, while AES spectra are usually complicated by a large number of background features. The current study focuses on the DAPS and omits allied threshold excitation techniques of the Auger Electron and Soft X-ray Appearance Potential Spectroscopies (AEAPS, SXAPS) because of the following reasons. DAPS data are considered enough to develop the methodology for a surface science study. Furthermore, AEAPS and SXAPS follow the core hole decay, which is inevitably complicated by the electron-core hole interaction. In contrast, the DAPS fixes the moment of core hole origination, when the electron-core hole interaction has not occurred or is minimal. The same is the case for photoelectron energy losses in XPS spectra, because the events of photon energy absorption and shakeoff/shake-up electron energy dissipation can proceed at once or shortly, eliminating or minimizing the electron-core hole interaction, respectively. 


\section{Conclusions}

The possibility to generate extra data by conventional techniques is truly desirable. This study highlights a rational model that gives a chance to realize this desire using ordinary XPS and DFT outputs. The model is based on the following statements. First, there are natural traps for the resonant energy absorption in the electronic configuration of atoms in a solid, such as shake-off, shake-up transitions, and plasmon oscillations; there is electronic affinity between the near-surface and bulk atoms. Second, the core level excitation of any origin forms multiple channels for the energy dissipation by filling those traps. Third, the relaxation can be traced experimentally, as the energy losses in XPS and DAPS spectra, and theoretically, via the convolution of the valence band DOS. The model does not conflict with general concepts of electron-solid interaction and has been well verified in model studies of Pt and graphite-based materials.

\section{Acknowledgements}

This study has been supported by the Russian Foundation for Basic Research (Grants 17-03-00049 and 16-03-00048). The authors thank D. V. Pinakov for the preparation of $\mathrm{C}_{2} \mathrm{~F}$ samples and Siberian supercomputer centre for granting their facilities for quantum-chemical calculations.

\section{Notes and references}

1 J. Sá, High resolution XAS/XES: analyzing electronic structures of catalysis, CRC Press, Boca Raton, 2014.

2 G. Bunker, Introduction to XAFS: A Practical Guide to X-ray Absorption Fine Structure Spectroscopy, Cambridge University Press, Cambridge, 2010.

3 L. Ament, M. van Veenendaal, T. Devereaux, J. Hill and J. van den Brink, Rev. Mod. Phys., 2011, 83, 705.

4 P. Brühwiler, O. Karis and N. Mårtensson, Rev. Mod. Phys., 2002, 74, 703.

5 A. Cholach and V. Tapilin, J. Chem. Phys., 2013, 138, 104201. 6 A. Cholach and V. Tapilin, Appl. Surf. Sci., 2001, 180, 173.

7 D. Briggs and M. Seach, Practical Surface Analysis by Auger and X-ray Photoelectron Spectroscopy, Wiley \& Sons, NY, 1983.

8 D. Woodruff and T. Delchar, Modern Techniques of Surface Science, Cambridge Univ. Press, Cambridge, 1994.

9 J. Rabalais, Principles of Ultraviolet Photoelectron Spectroscopy, Wiley, NY, 1977.

$10 \mathrm{~J}$. Kirschner, Electron-excited core level spectroscopes, Springer, Berlin, 1977.

11 G. Brodén, G. Briden and H. Bonzel, Surf. Sci., 1978, 72, 45.

12 CRC Handbook of Chemistry and Physics, ed. D. Lide, CRC Press, Boca Raton, 2005.

13 I. Asanov, A. Okotrub, A. Gusel'nikov, I. Yushina, D. Vyalikh and L. Bulusheva, Carbon, 2014, 82, 446.

14 I. Asanov, L. Bulusheva, M. Dubois, N. Yudanov, A. Alexeev, T. Makarova and A. V. Okotrub, Carbon, 2013, 59, 518.

15 N. Bausk, S. Erenburg, N. Yudanov and L. Mazalov, J. Struct. Chem., 1996, 37, 913.
16 P. Giannozzi, S. Baroni, N. Bonini, M. Calandra, R. Car, C. Cavazzoni, D. Ceresoli, G. L. Chiarotti, M. Cococcioni, I. Dabo, A. Dal Corso, S. de Gironcoli, S. Fabris, G. Fratesi, R. Gebauer, U. Gerstmann, C. Gougoussis, A. Kokalj, M. Lazzeri, L. Martin-Samos, N. Marzari, F. Mauri, R. Mazzarello, S. Paolini, A. Pasquarello, L. Paulatto, C. Sbraccia, S. Scandolo, G. Sclauzero, A. P. Seitsonen, A. Smogunov, P. Umari and R. M. Wentzcovitch, J. Phys.: Condens. Matter, 2009, 21, 395502. 17 J. Perdew, A. Ruzsinszky, G. Csonka, O. Vydrov, G. Scuseria, L. Constantin, X. Zhou and K. Burke, Phys. Rev. Lett., 2008, 100, 136406.

18 P. Blochl, Phys. Rev. B: Condens. Matter Mater. Phys., 1994, 50, 17953.

19 N. Marzari, D. Vanderbilt, A. De Vita and M. Payne, Phys. Rev. Lett., 1999, 8, 3296.

20 C.-H. Hu, P. Zhang, H.-Y. Liu, S.-Q. Wu, Y. Yang and Z.-Z. Zhu, J. Phys. Chem. C, 2013, 117, 3572.

21 A. Okotrub, N. Yudanov, I. Asanov, D. Vyalikh and L. Bulusheva, ACS Nano, 2013, 7, 65.

22 H. Monkhorst and J. Pack, Phys. Rev. B: Solid State, 1976, 13, 5188.

23 N. Ooi, A. Rairkar and J. Adams, Carbon, 2006, 44, 231.

24 O. Sedelnikova, L. Bulusheva and A. Okotrub, Synth. Met., 2010, 160, 1848.

25 H. Nejati and M. Dadsetani, Micron, 2014, 67, 30.

26 Supplement (Optimized atomic coordinates, DOS and views for the $\mathrm{C}_{24}, \mathrm{C}_{24} \mathrm{~F}_{12}$ and $\mathrm{C}_{24} \mathrm{~F}_{12} \mathrm{Br}_{2}$ unit cells).

27 J. Perdew, K. Burke and M. Ernzerhof, Phys. Rev. Lett., 1996, 77, 3865.

28 A. Rother and K. Scheerschmidt, Ultramicroscopy, 2009, 109, 154.

29 D. Gingrich, Practical Quantum Electrodynamics, CRC Press, NY, 2006.

30 C. Kittel, Introduction to solid state physics, Wiley, 2005.

31 B. Pennemann, K. Oster and K. Wandelt, Surf. Sci., 1991, 249, 35.

32 H. Bonzel and T. Fischer, Surf. Sci., 1975, 51, 213.

33 A. Ramstad, F. Strisland, S. Raaen, A. Borg and C. Berg, Surf. Sci., 1999, 440, 290.

34 M. Bartram, B. Koel and E. Carter, Surf. Sci., 1989, 219, 467. 35 J. Demuth, Surf. Sci., 1977, 65, 369.

36 A. Cholach, Chemical Physics Research Developments, Nova Sci. Publ., NY, 2011, pp. 149-173.

37 M. den Boer, T. Einstein, W. Elam, R. Park, L. Roelofs and G. Laramore, Phys. Rev. Lett., 1980, 44, 496.

38 E. Taft and H. Phillipp, Phys. Rev., 1965, 138, A197.

39 S. Tougaard and I. Chorkendorff, Phys. Rev. B: Condens. Matter Mater. Phys., 1987, 35, 6570.

40 L. Bulusheva, V. Tur, E. Fedorovskaya, I. Asanov, D. Pontiroli, M. Riccò and A. Okotrub, Carbon, 2014, 78, 137. 41 J. Feldman, W. Elam, A. Ehrlich, E. Skelton, D. Dominguez, D. Chung and F. Lytle, Phys. Rev. B: Condens. Matter Mater. Phys., 1986, 33, 7961.

42 O. Sedelnikova, L. Bulusheva, I. Asanov, I. Yushina and A. Okotrub, Appl. Phys. Lett., 2014, 104, 161905.

43 K.-D. Tsuei, J.-Y. Yuh, C.-T. Tzeng, R.-Y. Chu, S.-C. Chung and K.-L. Tsang, Phys. Rev. B: Condens. Matter Mater. Phys., 1997, 56, 15412. 